García, O. M. (2015). Geografía histórica e impacto ambiental del programa colonizador del Carare, Santander, Colombia 1953-1957. Revista Lebret, 7. Bucaramanga, Colombia: Universidad Santo Tomás, pp. 233 - 257. ISSN: 2145-5996.

\title{
Geografía histórica e impacto ambiental del programa colonizador del Carare, Santander, Colombia 1953-1957*
}

\author{
Historical geography and environmental impact program Carare \\ colonizer, Santander - Colombia 1953-1957
}

Olga Marina García Norato ${ }^{l}$

\begin{abstract}
Resumen
El artículo presenta a la comunidad académica el modelo novedoso de medición de impacto ambiental a partir de un programa colonizador, cuya construcción se logró por la pretensión de dar respuesta a la siguiente pregunta de investigación: ¿Contribuyó el programa de Colonización Agraria del periodo de gobierno 1953-1957 a ampliar la frontera agrícola del Carare, Santander en Colombia, de manera diferente a otras formas contemporáneas de colonización agraria? Dicho modelo se construyó mediante el apoyo de mapas oficiales del Instituto Geográfico Agustín Codazzi, se elaboraron mapas nuevos, que evidencian cambios en: recurso hídrico, carreteables, caminos construidos en vía, paisaje de construcción, límites geográficos y sitios poblados. Igualmente, aerofotografías tomadas antes, durante y después de la colonización, permitieron construir nuevos mapas y visualizar transformaciones en áreas de bosque, potrero, recurso hídrico y zona urbana para determinar las modificaciones que impactaron negativamente el sistema ambiental en la región.
\end{abstract}

Palabras clave

Impacto ambiental, mapas, aerofotografías, colonización, modelo.

\section{Códigos de clasificación JEL: Q1, Q2, Q5,013, P28, P42, N5}

\begin{abstract}
The purpose or this paper is to show to the academic community the innovative model for measuring environmental impact parting from a colonization program, which was constructed to answer this research question: Did the colonization agricultural program of the government period 1953-1957 contribute to extend the agricultural frontier of Carare - Santander in Colombia, in a way different from other contemporary manners or agricultural colonization? This model was constructed based on official maps of the Agustín Codazzi Geographical Institute (IGAC), new maps were developed which evidenced changes of scenery regarding: water resource, bridle paths, constructed and under construction roads, geographical boundaries and populated places. Identically, aerial photographs taken before, during and after colonization, allowed to construct new maps and to see transformations in forest, paddock, water and urban areas in order to determine modifications, which negatively impacted the environment of this region.
\end{abstract}

\section{Keywords}

Environmental impact, maps, aerial photographs, colonization, model.

* El artículo deriva del proyecto "Colonización Carare, Santander 1953-1957", de la tesis doctoral de la autora, auspiciado por el Departamento Nacional de Planeación de Colombia.

1 Doctorado en Historia Social y Económica. Docente investigadora del Centro de Investigación Louis Joseph Lebret de la Facultad de Economía de la Universidad Santo Tomás Bogotá, Colombia. Correo electrónico: olga. garcia@usantotomas.educ.co y olgarcia06@yahoo.es 


\section{Introducción}

La ampliación de las fronteras agrarias en Colombia durante el siglo XX y las trasformaciones naturales, fueron el resultado de las políticas de colonización agenciadas por el Estado desde inicios de siglo, ligadas particularmente a la construcción de obras públicas como carreteras y vías férreas, entre otras; además, a una intensa movilidad poblacional provocada por estas y por el auge cafetero y minero. Este proceso culminó con la colonización de vertiente y el inicio de la ampliación de frontera interior hacia zonas incultas ${ }^{2}$ del sur y el oriente del país, en las que se gestaron problemáticas económicas, sociales y políticas que pusieron un sello particular a los conflictos por la tierra y a las formas del poblamiento urbano. Estas transformaciones generaron cambios en la estructura de tenencia y uso de la tierra, desplazamientos, invasiones y alto impacto negativo en el medioambiente, acentuaron la deforestación devastadora y la degradación del paisaje natural y social, aceleraron el deterioro de los ecosistemas y provocaron cambios en la vocación económica de las regiones.

La iniciativa colonizadora sobre la región del Carare, por parte del Estado, se forjó mediante el Decreto 1894 de 1953, con el propósito de vincular a la economía nacional e internacional, la explotación económica de ricos y finos recursos maderables que por siglos se habían conservado en la región. Esta iniciativa colonizadora hizo parte, además, de la política de pacificación interna, teniendo como eje del proceso el retorno de campesinos desplazados y la promoción de la instalación de nuevos colonos en la región.

El presente artículo permite evidenciar los siguientes aspectos: primero el marco teórico (geografía histórica), segundo, el marco metodológico (modelo de medición), los resultados logrados especialmente en términos de la explicación geohistórica del programa colonizador y el análisis del impacto ambiental, finalmente, las conclusiones.

\section{Geografía histórica de la región del Carare}

Teniendo en cuenta los conceptos de geografía histórica planteados por diferentes autores cabe resaltar a Sauer (1925), quien la define como: "el estudio del cambio a través del tiempo, con un enfoque dinámico, donde los paisajes naturales se transforman lenta o rápidamente a través de la cultura". Por su parte Prince (1985) define la geografía histórica como "el análisis a través del tiempo de los cambios de tipo geográfico-espacial en el paisaje", explica que no posee un ámbito temático concreto, sino que sus conceptos y métodos se pueden aplicar a todos los campos de la geografía.

2 Este concepto era propio de los viajeros del siglo XVIII y XIX, cuestionado por ser su carga ideológica eurocéntrica y antropocéntrica... lo que no tenga la intervención de la "civilización" es "inculto". 
En Gran Bretaña, el auge de la geografía histórica, interesada por el espacio cuantitativo, produjo su propia crítica fructífera hasta encontrar textos donde se señala la importancia de la geografía histórica más allá de la propia geografía (Cortez, 1991). En México se han realizado escasos trabajos retomando los principios teóricos del espacio cuantitativo, sin embargo, en los trabajos de García (1998) se encuentra esta preocupación, en la que la clarificación de los hechos sociales están asociados a los aspectos funcionales a lo largo de la geografía, insertos también en el concepto de larga duración.

Cabe mencionar a Molano (2004), quien concibe la inserción histórica de la sociedad indígena en Villa de Leiva, Colombia, y evidencia cómo ese poblante americano antiguo que vivió en ese paisaje y vivió de él sin lesionarlo o alternándolo en mínimo grado, escribe lo que sucedió al irrumpir allí la humanidad hispánica con sus propias instituciones políticas, económicas y sociales, reseña este tramo del acontecer geográfico durante los tiempos coloniales. Y termina señalándonos cuál es la realidad villaleivana en los siglos XIX y XX.

Otro autor que se destaca por sus investigaciones en Chile y Venezuela es Cunill (2009), quien presenta en su prolífica obra geohistórica, numerosos trabajos cuya periodización se desarrolla desde el siglo XVI, y se detiene de preferencia en el XIX, pasado al que penetramos a través del estudio de la población y la manera como ella dispuso de los recursos, creando y destruyendo paisajes, además, plantea la importancia del espacio en la evolución geohistórica latinoamericana o, dicho de otro modo, la manera cómo el espacio físico y las cambiantes situaciones ambientales han condicionado a las sociedades humanas en su estructuración paisajística.

En la región del Carare, este artículo presenta los cambios de paisaje generados por el programa colonizador orientado en el Gobierno 1953-1957, está localizada en la zona suroccidental del departamento de Santander, que se ubica en el noreste de Colombia. Santander era uno de los departamentos más montañosos y, además, poseía una amplia zona plana y baja, por lo cual se pueden distinguir dos grandes unidades fisiográficas: el valle del Magdalena y la cordillera Oriental.

\section{El Carare en el valle del Magdalena}

Una parte de la región del Carare se extiende hasta el valle del Magdalena Medio, el cual se encuentra ubicado en la mitad occidental del departamento de Santander; su topografía se caracteriza por ser plana y suavemente ondulada, "con predominio de acumulaciones fluviales del Cuaternario y rocas sedimentarias del Terciario. La lluvia es del orden de 2500 - $3000 \mathrm{~mm}$ anuales, con leve incremento en los alrededores de Barrancabermeja" (IGAC, 1989, p. 361). En la parte occidental, sobre la ribera del río Magdalena, predomina la vegetación selvática, y hacia el centro oriente, el bosque ecuatorial, ambos tipos de vegetación, del piso bioclimático cálido. Los suelos son pobres, aptos para las actividades ganaderas. 


\section{La cordillera Oriental}

La cordillera Oriental recorre el costado oriental del departamento, en dirección general suroeste-noreste.

Es de topografía quebrada y abrupta, con alturas superiores a $3000 \mathrm{msnm}$, como en la cordillera de los Yariguíes, divisoria fluvial, río Suárez al oriente y río Magdalena al occidente: las lluvias en la mayor parte de la cordillera son de 1500 - 2000 mm" (IGAC, 1989, p. 361).

La vegetación corresponde a los pisos bioclimáticos templados y fríos. Los suelos, desarrollados en laderas, son pobres en bases, aptos para uso agrícola selectivo y para cobertura vegetal. Es uno de los tres ramales en los que se divide la cordillera de los Andes en Colombia, parte del macizo colombiano en dirección norte, la región occidental de la cordillera Oriental pertenece a la cuenca del río Magdalena.

\section{El proyecto de colonización militar en el Carare, Santander, Colombia. Decreto 0870 del 26 de marzo de 1953}

A partir del Decreto 0870, expedido el 26 de marzo de 1953, firmado por el designado presidente Roberto Urdaneta Arbeláez, quien reemplazaba temporalmente al presidente Laureano Gómez, dos meses y medio antes de asumir como presidente de la República de Colombia el general Gustavo Rojas Pinilla, quien era el comandante de las Fuerzas Armadas de Colombia, se asignan dos zonas de colonización militar en el valle del río Magdalena, las cuales se evidencian en la construcción del mapa 1. La institución que asumió inicialmente la responsabilidad social y económica de la colonización fue el Instituto de Parcelaciones, Colonización y Defensa Forestal, una vez asume la presidencia el general Gustavo Rojas Pinilla, crea el Instituto de Colonización e Inmigración, el cual, en coordinación con los ministerios de Guerra, Agricultura, Higiene y Obras Públicas, el Instituto de Crédito Territorial, Aeronáutica Civil y la Caja de Crédito Agrario Industrial y Minero, entre otras, debía coordinar la titulación de baldíos, del Valle del río Magdalena, ubicadas en los departamentos de Santander y Antioquia, y la presencia institucional para la dotación de infraestructura de apoyo a la producción y reproducción social de la población colonizadora, como se evidenció en las dos décadas siguientes de la ejecución del programa, con la creación de municipios como: Cimitarra, Landázuri, Puerto Parra y Santa Helena del Opón.

\section{Primera zona}

Ubicada en el departamento de Santander (de color verde en el mapa 1); de acuerdo con el Decreto mencionado, estaba delimitada así: 
Partiendo de la desembocadura del río Carare, en el río Magdalena, se sigue aguas abajo por este hasta encontrar la desembocadura del río Opón, en el brazo Opón; de este punto aguas arriba hasta encontrar la desembocadura de la quebrada negra de Armas, en este río; se sigue por la quebrada Negra de Armas aguas arriba hasta su nacimiento; de aquí en línea recta al suroeste hasta encontrar el río Horta, cerca al lugar denominado Rancho Grande; siguiendo por el río Horta Aguas abajo hasta su desembocadura del río Carare o Minero; de este sitio aguas abajo del río Carare hasta su desembocadura en el río Magdalena, punto de partida (Presidencia de la República, 1953).

En la delimitación anterior se ubica la región del Carare, Santander, Colombia, objeto de la investigación y área utilizada para la construcción del modelo de medición de impacto ambiental presentado en este artículo. Rojas Pinilla asume funciones presidenciales el 13 de junio de 1953, y en el mes de julio del mismo año creó el Instituto de Colonización e Inmigración, en reemplazo del Instituto de Parcelaciones Colonización y Defensa Forestal; la nueva institucionalidad asumió el liderazgo en el programa de colonización de esta zona.

\section{Segunda zona}

Cubría de manera general las tierras servidas por la margen occidental del río Magdalena, identificadas de color azul en el mapa 1, se encuentra ubicada en el departamento de Antioquia, fuera del área objeto de estudio.

\section{Mapa 1. Zonas de colonización militar en el valle del río Magdalena}

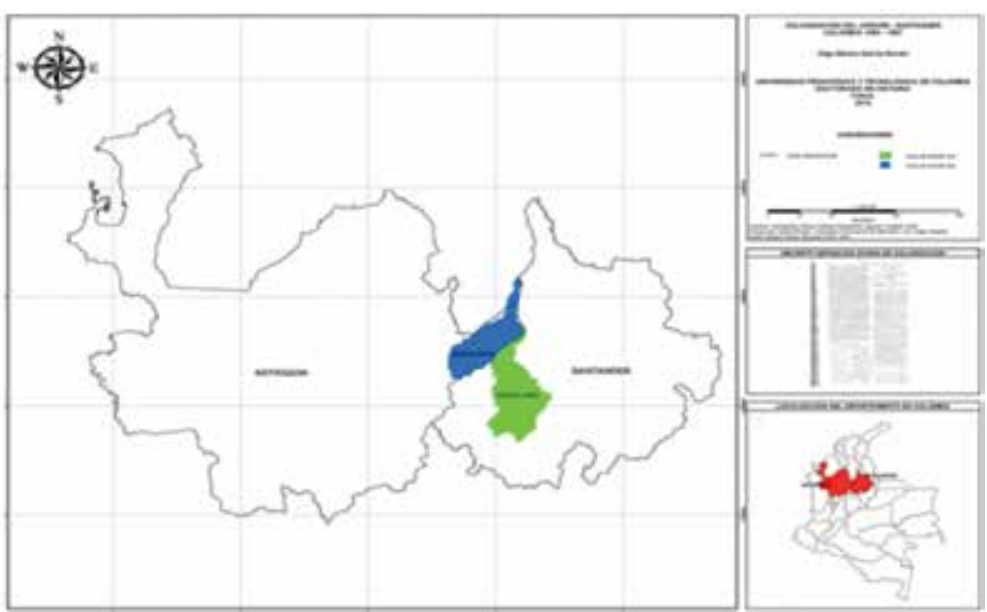

Fuente: Autora. Colonización Militar Carare, Santander, Colombia. Con base en el Decreto 870 del 26 de marzo de 1953, ubicación en la cartografía oficial del IGAC, 2008. Proyección Gauss - Kruger, (Transversal de Mercator), origen Bogotá. Dantum. Magna - Sirgas Elipsoide GRS 1980. Tunja, agosto, 2012. 


\section{Metodología del modelo de medición de impacto ambiental a partir de la colonización del Carare, Santander, Colombia}

Para identificar y caracterizar las transformaciones inducidas por el proceso colonizador en la región, se recurrió al análisis e interpretación de las únicas cartas geográficas del departamento de Santander, conservadas en custodia por el Instituto Geográfico Agustín Codazzi (IGAC) de Colombia, correspondientes a los años 1941, 1952 y 1972. Estas se utilizaron como referente para situar la zona del Carare, teniendo en cuenta las coordenadas del IGAC, y como resultado de ese ejercicio analítico se produjeron tres nuevos mapas, de la región priorizada por el general Rojas Pinilla, para establecer allí el programa de colonización durante los dos primeros años de su periodo de gobierno (1953-1957).

A través de los nuevos mapas construidos como resultado de esta investigación, fue posible evidenciar cambios de paisaje en los siguientes aspectos: recurso hídrico, carreteables, caminos construidos, en vía de construcción, límites geográficos y sitios poblados de la región; cabe subrayar que la pretensión de este artículo, es mostrar a partir de esta caracterización los cambios generados por la colonización, los cuales se observan en la construcción del nuevo mapa de 1972.

Igualmente, se seleccionaron tres aerofotografías de vuelos oficiales realizados por personal del IGAC, correspondientes a los años 1950, 1957 y 1977. Se efectuó un ejercicio de fotointerpretación, que permitió establecer, con el apoyo y uso del software ArcGIS Versión 9.3, el impacto ambiental generado por el proceso de colonización dirigida por el gobierno Rojas Pinilla. De este análisis fue posible construir nuevos mapas, que permitieron medir y visualizar gráficamente los cambios de paisaje en áreas de bosque, potrero, recurso hídrico y sitios poblados, y así determinar los efectos que impactaron negativamente el sistema eco-ambiental en la región colonizada.

Al llevar las tres aerofotografías seleccionadas a escala 1:10.000, para hacer compatible la comparación y medición con los datos evidenciados en la fotografía tomada en 1977, y así adoptar un método riguroso de superposición y comparación, se seleccionó el sitio de ubicación del asentamiento poblacional del hoy municipio de Cimitarra, sede del programa oficial de Colonización del Carare. Como resultado de este proceso fue posible medir las siguientes áreas: boscosa, potrero, hídrica y sitios poblados, en las tres épocas comparadas, para determinar con alto grado de exactitud, el profundo impacto ambiental negativo que generó la empresa colonizadora en la región del Carare.

\section{Cartas geográficas del departamento de Santander 1941, 1952 y 1972}

Las únicas cartas para realizar la caracterización de la zona de colonización del Carare se ubicaron en el IGAC y corresponden a los años 1941, 1952 y 1972. Estas cartas se utilizaron con el fin de alcanzar la producción de tres nuevos mapas, en 
los que se analiza la región priorizada por el general Rojas Pinilla para establecer el programa de Colonización del periodo 1953-1957; los mapas nuevos demuestran cambios de paisaje, de recurso hídrico, carreteables, caminos construidos, en vía de construcción, límites geográficos y sitios poblacionales de la región.

\section{Departamento de Santander, Colombia 1941 (mapa 2)}

La información suministrada por el IGAC, correspondiente al departamento de Santander en 1941, presenta las siguientes características:

(...) posiciones astronómicas fijadas por la oficina de longitudes para los pormenores topográficos, se han utilizado algunos planos especiales, de los ríos navegables, itinerarios e intersecciones de la misma oficina y la nomenclatura de los nuevos croquis municipales. Se ha usado para esta carta la proyección sinusoidal, superficie de 32070 kilómetros cuadrados. Coordenadas de Bogotá: 4 grados, 35 minutos, 56 segundos al Norte. Latitud de 74 grados, 4 minutos, 51 segundos al Oeste y una altura de 2640 metros sobre el nivel del mar (IGAC, 1941).

\section{Mapa 2. Carta geográfica del departamento de Santander}

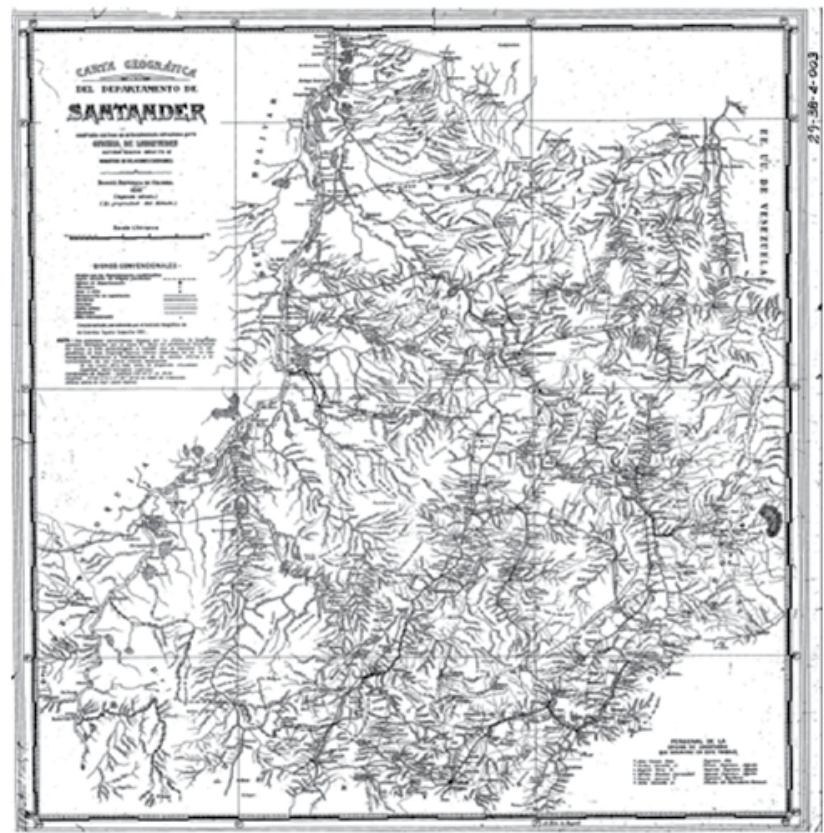

Fuente: IGAC, mapa físico-político de Colombia. Cartografía oficial Instituto Geográfico Agustín Codazzi. Proyección Gauss - Kruger, Colombia (Transversal de Mercator), con origen Bogotá, 1980, escala 1:6.000.000. 
Región Carare, Santander, Colombia 1941, escala 1:500.000. Cambios de paisaje: recurso hídrico, carreteables, caminos construidos, en vía de construcción, límites geográficos y sitios poblados (mapa 3)

Con base en el mapa 2, de 1941, se construyó el mapa 3 donde se localiza la zona del Carare, Santander, Colombia, se caracteriza la región en términos geográficos, hídricos, ferrocarriles, caminos y carreteables, se identifica y determinan las condiciones en las cuales se encontraba antes del proceso de Colonización orientado en el gobierno de Rojas Pinilla. En la delimitación de la zona de estudio para 1941 se aprecia el curso de la cuenca y las micro cuencas de los ríos Carare y Opón, así como la vía carreteable que comunica de occidente a oriente a Puerto Berrío con el sitio poblacional de Landázuri; que para la época no tenía una delimitación territorial del municipio como tampoco el hoy municipio de Cimitarra (mapa 3).

Carreteables. Teniendo en cuenta que para 1941 el Estado colombiano no había hecho presencia en la región del Carare, no se evidencian zonas urbanas; solamente se observa un carreteable que partía de Vélez en dirección noroccidente a encontrar la quebrada Unión, continua por la margen izquierda del río Guayabito, hasta encontrar el río Carare, donde el carreteable desvía hacia el occidente. Se aprecia un camino de herradura que parte del río Opón hacia el suroccidente, hasta la intersección de las quebradas María y Verde, donde termina.

Recurso hídrico. En 1941 la región del Carare ostentaba una extraordinaria vegetación y red hídrica; se destacan el río Opón, que recorre la zona por el oriente y cuya hoya hidrográfica la componen tres ríos: San José, Guayabo y Meritosa y ocho quebradas: Portones, Presidente, Bogie, Loro, India, Macía, Los Muertos y Caño Achi; el río Horta, que hace su recorrido por el sur de la zona para unirse con el río Minero y dar origen al río Carare, al cual tributan las siguientes quebradas: La India, Vinagre, Santa Rosa, Cabo Plata, Torura, Los Botes, Caraverde, Corroverde, Caño Porquería, Mula, Caño del Cerro, Caño Rabón y La Negra, y el río Guayabito, que atraviesa por el centro de la zona del Carare, mostrando un hermoso paisaje. En cuanto a ciénagas, se observan las siguientes: Chucurí, Chara, Porquería y Rompida. Los cerros que se destacan son el cerro de Armas y la cordillera Montellina, donde nace el río Horta. En este mapa no se aprecian zonas urbanas.

Sitios $^{3}$. En el mapa de 1941 se observan muy pocos sitios, debido a la baja infraestructura vial y de servicios, por la poca o nula presencia institucional, lo cual permite inferir la menguada población asentada en la cuenca de los ríos Carare, Opón y Guayabito. Cabe destacar que los sitios ubicados sobre la cuenca del río Carare son: Fortuna, Santa Rosa, Raizuelo, San Fernando y Esmeralda; sobre la cuenca del río Opón se encuentran el sitio denominado Portones, y al suroriente de la región del Carare, sobre el río Guayabito, se encuentra el sitio llamado Lugo.

3 Sitio: término utilizado por la O ficina de Longitudes, entidad té c nica a dscrita en 1941 al Ministerio de Relaciones Exteriores, Bogotá, Colombia. 
Mapa 3. Región del Carare, Santander, Colombia 1941, escala 1:500.000

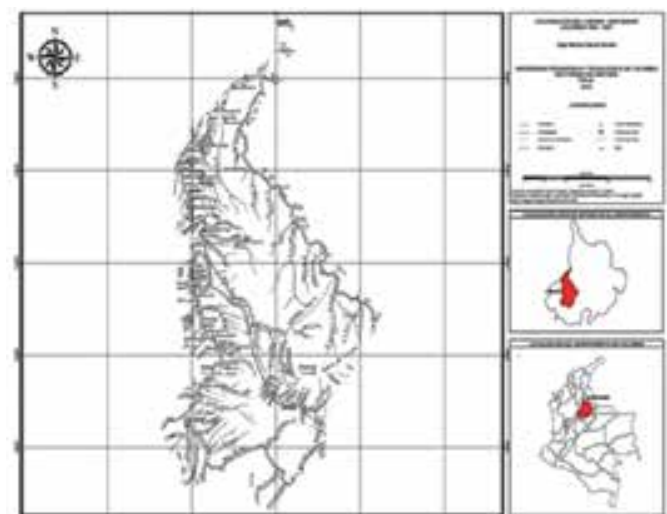

Fuente: Autora. Región Carare, Santander, Colombia 1941, escala 1:500.000, elaborado con base en el mapa 2 sobre las coordenadas oficiales del IGAC. Tunja, agosto, 2012.

\section{Departamento de Santander, Colombia 1952, escala 1:300.000 (mapa 4)}

La información suministrada por el IGAC, correspondiente al departamento de Santander en 1952 muestra los mismos límites que el mapa de 1941, y se describen a continuación los siguientes aspectos: carreteables, recurso hídrico y sitios.

Mapa 4. Santander, Colombia 1952, escala 1:300.000

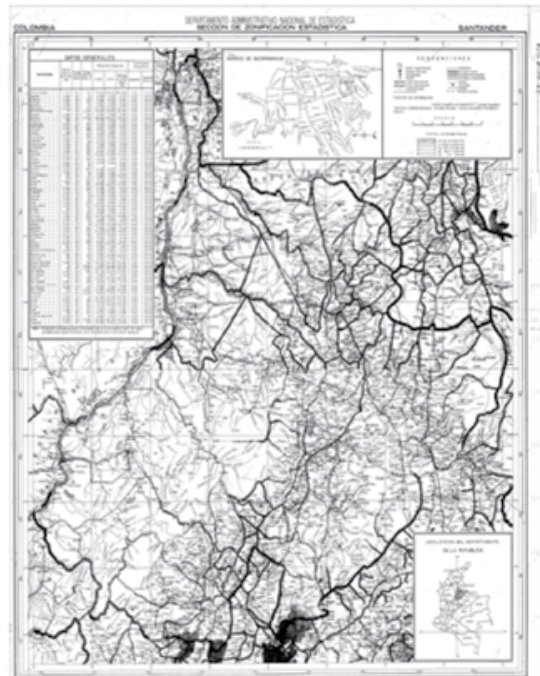

Fuente: Departamento Administrativo Nacional de Estadística (DANE). Santander, Colombia 1952, escala 1:300.000. “A.C.” Sociedad Geográfica Americana - Compañías petroleras, entidades oficiales, Archivo Cartográfico Estadística Nacional, 1952. 
Región del Carare - Santander - Colombia 1952, escala 1:300.000. Cambios de paisaje: recurso hídrico, carreteables, caminos construidos, en vía de construcción, límites geográficos y sitios poblados (mapa 5)

Carreteables. Se evidencia en el mapa 5 que la dotación de infraestructura vial de la zona del Carare en 1952 presenta nuevas vías, debido al proceso de industrialización del país; uno de los propósitos de ampliación de las vías fue permitir el transporte de los equipos que se importaron con destino a la construcción de Acerías Paz del Río, S. A., la cual nació en 1948, por iniciativa del Gobierno colombiano, bajo el nombre de «Empresa Siderúrgica Nacional de Paz de Río", y el 17 de septiembre del mismo año inició la explotación de las minas de hierro y carbón en Boyacá, así como la construcción de la primera planta siderúrgica con alto horno y laminación en el país, en los terrenos de la antigua Hacienda Belencito, en el municipio de Nobsa, Boyacá, donde se ha mantenido hasta la actualidad. Se presume que por esta razón el carreteable visto en el mapa 5 del año 1952, que comunica las poblaciones de Landázuri, Cimitarra y Puerto Araujo se ha convertido en carretera. Aparece una nueva vía, que va de Santa Rosa (Carare) a la vía que conduce de Cimitarra a Puerto Araujo.

Aparecen nuevos caminos de herradura, tales como: en el suroriente de la zona se encuentra el camino que comunica los cerros Las Armas y San Ignacio del Opón; hacia el suroccidente de la región del Carare aparece el camino de herradura que conduce del sitio denominado Brasil a la población de Cimitarra, y otro camino, que va de la cordillera Sabana Alta a interceptar el camino Brasil-Cimitarra. En el municipio de Cimitarra aparece un camino que conduce de Garcés a Puerto Parra, el cual comunica a la región denominada Ley a Toyó. Igualmente, se puede observar el ferrocarril que conduce de Puerto Olaya a Barrancabermeja, y el que conduce del sitio Las Mulas hacia el río Opón.

Mapa 5. Región del Carare, Santander, Colombia 1952, escala 1:300.000

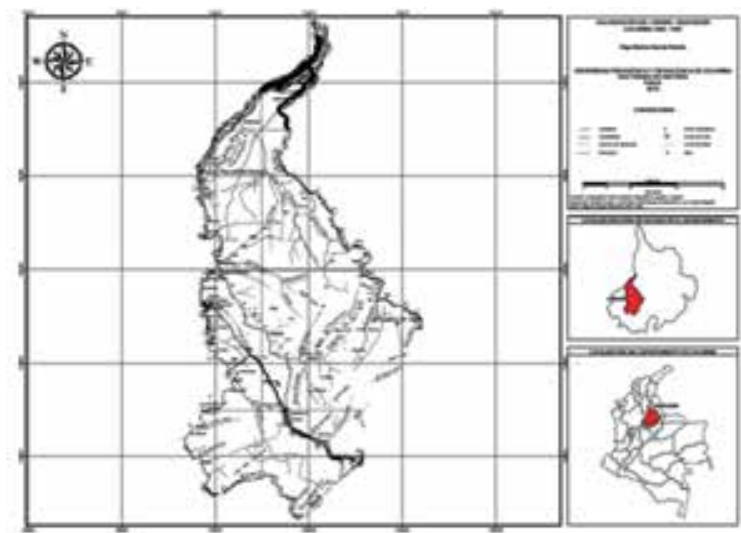

Fuente: Autora. Región Carare, Santander, Colombia 1952, escala 1:300.000 a partir del mapa 4 sobre las coordenadas oficiales del IGAC. Tunja, agosto, 2012. 
Recurso hídrico. El recurso hídrico de la zona del Carare en 1952, observado en el mapa, disminuye; se destaca la presencia del río Blanco en la cuenca del río Opón, al igual que las quebradas San José y La India; se evidencia que han desaparecido 6 quebradas y dos ríos en relación con la descripción de este recurso relacionada en el mapa de 1941. Sobre el río Carare se observan las quebradas Quebradona, Santa Rosa y Parra.

Sitios. Es importante destacar que en el mapa de 1952 han desaparecido o cambiado de nombre algunos sitios que se observaron en el mapa de 1941, y que aparecen nuevos caseríos ${ }^{4}$, como Landázuri, Cimitarra, Puerto Araujo, Puerto Parra (Puerto Carare), Chucurí y el sitio denominado San Ignacio del Opón. Esto obedece a la ampliación de las vías y carreteables mencionados anteriormente, lo cual permite relacionar estos caseríos con presencia de nuevos pobladores en la región del Carare.

\section{Santander, Colombia 1972, escala 1:500.000 (mapa 6)}

El mapa de Santander, Colombia de 1972 fue seleccionado para el análisis de la situación tras finalizar el periodo de Gobierno 1953-1957, durante el cual se hizo presencia institucional en la región del Carare, con el propósito de ejecutar el programa de colonización. Con la información contemplada en el mapa del departamento de Santander de 1972 se evidencian los cambios generados en carreteables, recurso hídrico y municipios; estos cambios se pueden apreciar en el mapa 7 con mayor claridad.

Mapa 6. Mapa Santander, Colombia 1972, escala 1:500.000

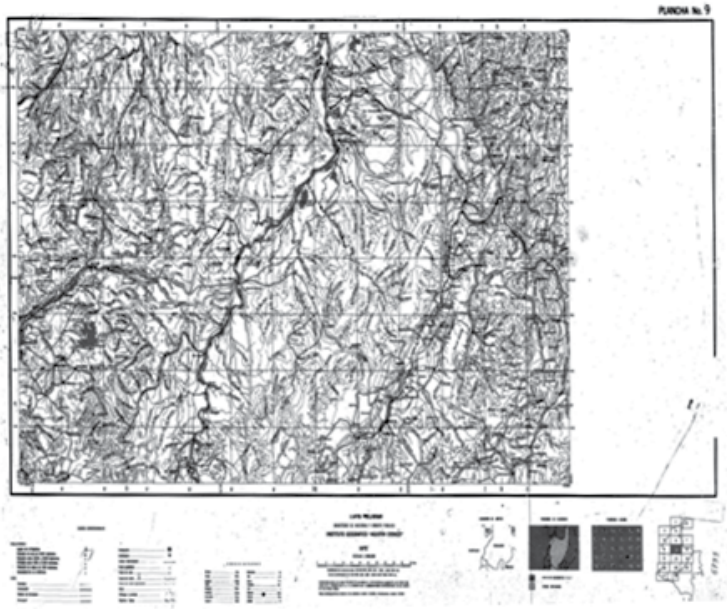

Fuente: Carta preliminar Ministerio de Hacienda y Crédito Público. IGAC. Bogotá, Colombia, 1972.

4 Caserío (CAS). Sitio que presenta un conglomerado de viviendas, ubicado comúnmente al lado de una vía principal y que no tiene autoridad civil. El límite censal está definido por las mismas viviendas que constituyen el conglomerado. DANE. División Político Administrativa de Colombia, 1954. 
Región del Carare, Santander, Colombia, 1972, escala 1:500.000. Cambios de paisaje: recurso hídrico, carreteables, caminos construidos, en vía de construcción, límites geográficos y sitios poblados (mapa 7)

Carreteables. Teniendo en cuenta la presencia institucional en la región del Carare durante el periodo 1953-1957, liderada por el Instituto de Colonización e Inmigración para ejecutar el programa de colonización, es pertinente señalar que los ministerios de Obras Públicas, Higiene, Educación, Hacienda, Industria y Comercio, Agricultura y Defensa, y el Instituto de Crédito Territorial (INSCREDIAL), la Caja de Crédito Agrario Industrial y Minero, el Banco Popular y la Aeronáutica Civil, participaron en la dotación de infraestructura vial y de servicios en dicho periodo de gobierno, lo cual generó cambios importantes en el paisaje observado en el nuevo mapa 7 .

Así las cosas, el carreteable que en 1952 conducía del sitio Brasil al sitio Cimitarra, se convirtió en una carretera pavimentada; igualmente, la carretera entre los sitios Landázuri - Cimitarra - Puerto Parra se encuentra pavimentada; en cambio, el carreteable que conducía de Santa Rosa a la central Cimitarra - Puerto Parra se visualiza sin pavimentar. Se observa el aeródromo ubicado en el municipio de Cimitarra, el cual fue construido e inaugurado en 1954 como producto del programa de Colonización, en aras de conseguir el progreso de la región del Carare a partir de la dotación de infraestructura vial construida en el periodo de gobierno del general Gustavo Rojas Pinilla, y a partir del programa de Colonización dirigida se pretendió vincular el potencial maderero de la región a la economía nacional e internacional.

Se observa en el mapa 7, el ferrocarril que conduce de Puerto Olaya a Barrancabermeja, el cual pertenecía al que unía a Bogotá con la Costa Atlántica; además, se destaca que desapareció el ferrocarril que conducía del sitio denominado Las Mulas hacia el río Opón. Los caminos de herradura desaparecieron en este mapa, correspondiente al año 1972. Finalmente, cabe destacar en el mapa el oleoducto que va de Puerto Berrío a Barrancabermeja, toda vez que la región es rica en producción de petróleo, el cual es transportado a la refinería de Barrancabermeja. Algunos de los efectos negativos generados a partir de la construcción de las vías en la región del Carare, hacen referencia al impacto drástico del medio biofísico, socioeconómico y cultural de su entorno.

Recurso hídrico. Lamentablemente, el recurso hídrico disminuyó debido a la reducción sensible del área de bosque, convertida en potreros, como producto de la colonización; es por ello que en la cuenca del río Opón solo se observa la quebrada La India y el Río Blanco. Sobre la cuenca del río Carare quedaron únicamente los ríos Guayabito y Toroba y las quebradas Agosto, Montoyas y Cristóbal. Cabe destacar que desaparecieron las ciénagas de Chara, Porquería y Rompida; solo quedó la ciénaga de Chucuri. 
Municipios y poblaciones. En la división política administrativa del departamento de Santander, la región del Carare correspondía al territorio del municipio de Vélez. A partir del desarrollo del programa de colonización se generaron cambios poblacionales que permitieron crear los siguientes municipios: Cimitarra, Santa Helena del Opón, Landázuri y Puerto Parra entre otros.

Mapa 7. Región del Carare, Santander, Colombia, 1972, escala 1:500.000

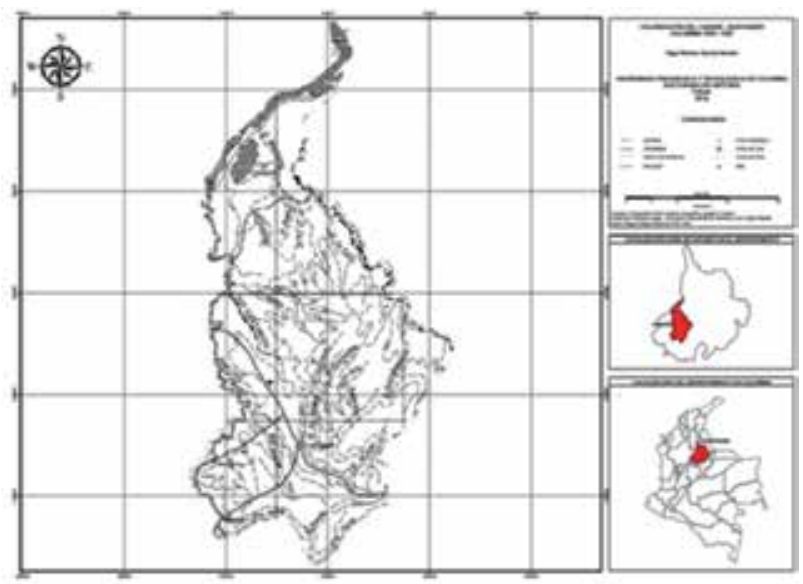

Fuente: Autora. Región del Carare, Santander, Colombia 1972, escala 1: 300.000, elaborado con base en el mapa 6 sobre las coordenadas oficiales del IGAC. Tunja, agosto, 2012.

\section{Ocupación y uso del suelo en la región del Carare vista en aerofotografías de 1950, 1957 y 1977}

Para establecer la dinámica de ocupación y uso del suelo en la región de colonización del Carare, la investigación se apoyó en el método de fotointerpretación de aerofotografías oficiales hechas por el IGAC para 1950, 1957 y 1977. Una vez adelantada la revisión de las aerofotografías se seleccionaron tres, con el propósito de efectuar la fotointerpretación y, a partir de ella, utilizando el software ArcGIS Versión 9. 3, determinar, en tres épocas diferentes, el impacto ambiental del programa de colonización dirigida. Mediante esta técnica fue posible medir y establecer las áreas de bosque, potrero, recurso hídrico y zona o sitios poblados, lo cual facilitó identificar los cambios que impactaron negativamente el sistema ambiental en la región colonizada.

Aerofotografía año 1950, región del Carare, Santander, Colombia, escala $1: 30.000$

Condiciones geoestructurales de la Región de colonización del Carare, Santander. En la aerofotografía de 1950, hecha a escala 1:30.000, antes de la implementación 
del programa de colonización sobre la región del Carare, es posible apreciar una uniformidad topográfica, característica de los bosques andinos nativos, con unas trazas insignificantes de asentamientos poblacionales aislados y dispersos.

Aerofotografía 1. Cimitarra, Santander, Colombia, 1950

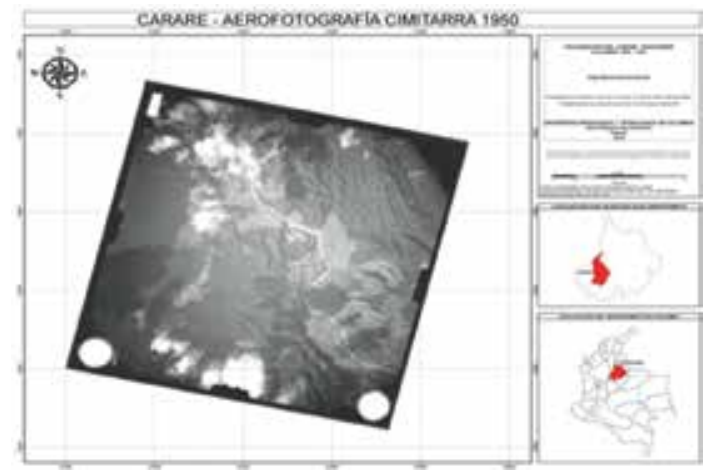

Fuente: Aerofotografía pancromática C-584 No. 172 escala 1:30.000. IGAC, Proyección Gauss -

Kruger, Colombia (Transversal de Mercator), con origen Bogotá, 1980.

Aerofotografía año 1957, región del Carare, Santander, Colombia, escala 1:20.000

A partir de la aerofotografía de 1957 pancromática R-431 No. 751 a escala 1:20.000 de 1957 en formato digital, fotográficamente con resolución de quince (15) micrones en formato TIFF., correspondiente a la región del Carare, tomada del archivo histórico del IGAC, la cual tiene un área inferior a la tomada en 1950 por su escala, se realizó la fotointerpretación, utilizando el software ArcGIS, versión 9.3.

Aerofotografía 2. Cimitarra, Santander, Colombia, 1957

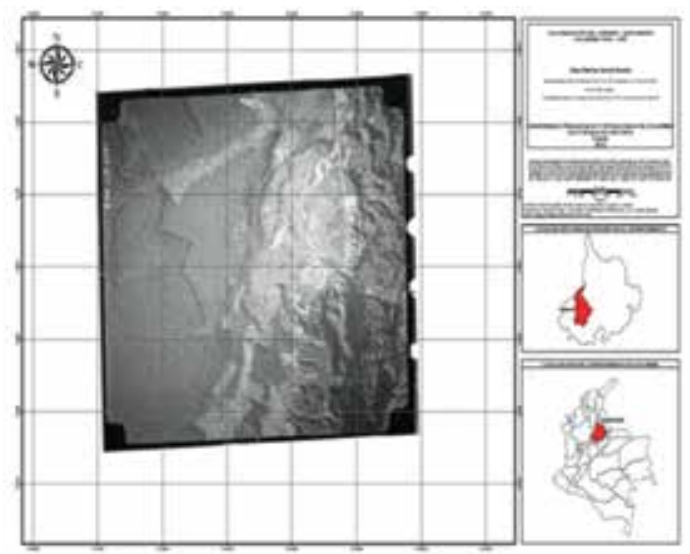

Fuente: Aerofotografía pancromática R-431 No. 751 escala 1:20.000 de 1957. IGAC, Proyección Gauss - Kruger, Colombia (Transversal de Mercator), con origen Bogotá, 1980. 
Aerofotografía año 1977 Región del Carare, Santander, Colombia, escala 1:10.000

Esta aerofotografía que es posterior al periodo de estudio, constituye un referente que permite comparar y contrastar los estados y los cambios operados en la zona producto de la empresa colonizadora.

Se puede apreciar claramente el cambio de paisaje, de bosque a potrero, y el crecimiento de la zona urbana de Cimitarra, que, una vez cumplió con los requisitos de población y dotación de servicios, fue erigido municipio en 1966. Se puede demostrar con base en esta aerofotografía de 1977, los efectos del programa Colonizador en la región del Carare; así como otros efectos producidos por la colonización, tales como el desarrollo de Landázuri, Santa Helena del Opón y Puerto Parra, que también generaron posibilidades de crecimiento poblacional y de infraestructura en la región.

Aerofotografía 3. Cimitarra, Santander, Colombia, 1977

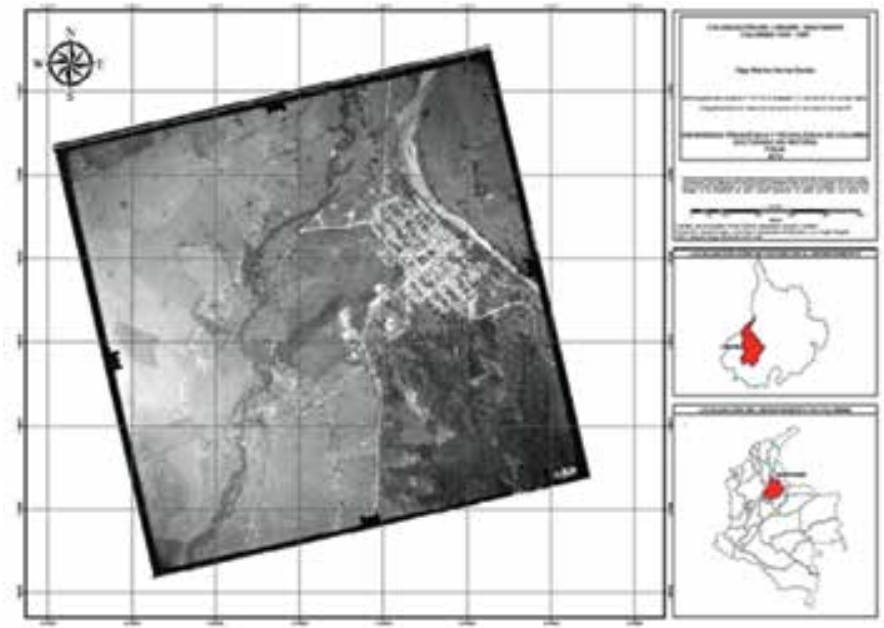

Fuente: Aerofotografía pancromática C-1757 No. 00 escala 1:10.000 de 1977. IGAC, Proyección Gauss Kruger, Colombia (Transversal de Mercator), con origen Bogotá, 1980.

\section{Tendencias de los cambios físicos naturales del paisaje en la zona de colonización del Carare}

Con el objeto de demostrar técnicamente la ampliación de la frontera agrícola, los cambios físicos y el impacto ambiental negativo en la región del Carare, específicamente sobre el área de ubicación del centro colonizador en Cimitarra, se realizó un análisis a través de la superposición de las aerofotografías de los años 1950, 1957 y 1977, a escala 1:10.000 y se logró el siguiente resultado: 


\section{Condiciones naturales de la región de Colonización en 1950. Cimitarra, Santander, Colombia, escala 1:10.000}

Para apreciar las condiciones naturales de la región de colonización del Carare, nos apoyamos en la aerofotografía de 1950 a escala 1:30.000, tomada sobre la el municipio de Cimitarra y convertida a escala 1:10.000, a continuación se presenta la interpretación de la aerofotografía: se evidencia en la figura 1 y la tabla 1, el área que comprende vegetación boscosa, marcada en la figura 1 con el color verde oscuro, cubre el 73,89\% del área total, mientras que los pastizales, marcados con color verde claro, cubren el $18,5 \%$ y el recurso hídrico delineado con color azul corresponde al $7.6 \%$.

Tabla 1. Ocupación - uso del suelo según aerofotografía de 1950 a escala 1:10.000, Cimitarra - Carare, Santander, Colombia

\begin{tabular}{|l|c|c|}
\cline { 2 - 3 } \multicolumn{1}{c|}{} & Área $(\mathrm{m} 2)$ & $\%$ \\
\hline Bosque & 4845295,20 & 73,89 \\
\hline Potrero (pastizales) & 1213130,41 & 18,50 \\
\hline Recurso hídrico & 498991,91 & 7,61 \\
\hline Total & 6557417,52 & 100 \\
\hline
\end{tabular}

Fuente: Autora. Ocupación - uso del suelo según aerofotografía de 1950 a escala 1:10.000, Cimitarra Carare, Santander, Colombia a partir de la interpretación de la aerofotografía pancromática C-584 No. 172 escala 1:30.000. IGAC, Proyección Gauss - Kruger, Colombia (Transversal de Mercator), con origen Bogotá, 1980. Tunja, agosto, 2012.

Figura 1. Cobertura uso del suelo Cimitarra - Carare, Santander, Colombia, 1950

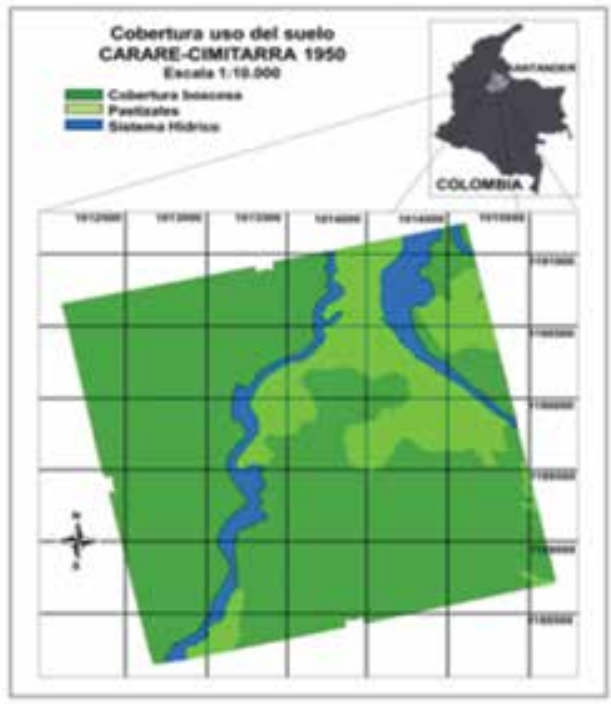

Fuente: Autora. Cobertura uso del suelo Cimitarra - Carare, Santander, Colombia, 1950, a partir de la aerofotografía pancromática C-584 No. 172 escala 1:30.000 de 1950. IGAC, Proyección Gauss - Kruger,

Colombia (Transversal de Mercator), con origen Bogotá, 1980. Tunja, agosto, 2012. 


\section{Condiciones naturales de la región de Colonización en 1957. Cimitarra, Santander, Colombia, escala 1:10.000}

Se pueden evidenciar en la tabla 2 y la figura 2 los cambios de paisaje generados como resultados del programa de colonización, grandes cambios como producto de la colonización, ya que la cobertura boscosa que se marca en la figura 2 con tonalidad verde oscuro, y se distribuye en un $32,17 \%$ del total de la imagen, refleja la pérdida del 41,72\% del área de bosque, en el transcurso de 1950 a 1957; simultáneamente, las áreas de entresaca medianamente conservadas se muestran de color verde oliva, y los pastizales con color verde claro; incrementan sus áreas en 30,14\% y 30,35\%, respectivamente, a las estimadas en 1950.

Tabla 2. Ocupación, uso del suelo según aerofotografía de 1957 a escala 1:10.000, Cimitarra - Carare, Santander, Colombia

\begin{tabular}{|l|c|c|}
\cline { 2 - 3 } \multicolumn{1}{c|}{} & Área $(\mathrm{m} 2)$ & $\%$ \\
\hline Bosque & 2109626,60 & 32,17 \\
\hline Bosque medianamente conservado & 1976340,66 & 30,14 \\
\hline Potrero (pastizales) & 1990267,42 & 30,35 \\
\hline Recurso hídrico & 382902,49 & 5,84 \\
\hline Área Urbana & 98280,36 & 1,50 \\
\hline Total & 6557417,53 & 100 \\
\hline
\end{tabular}

Fuente: Autora, con base en los datos de la aerofotografía de 1957, a escala 1:10.000. Tunja, agosto, 2012.

Figura 2. Cobertura uso del suelo Cimitarra - Carare, Santander, Colombia, 1957

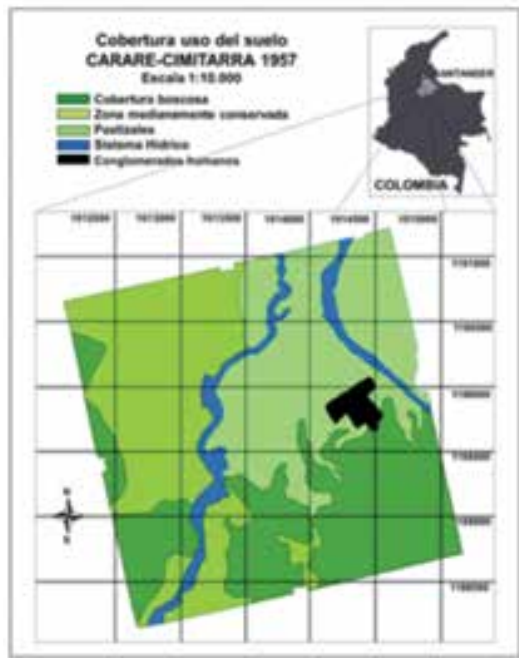

Fuente: Autora. Cobertura uso del suelo Cimitarra - Carare, Santander, Colombia 1957, a escala 1:10.000, con base en aerofotografía pancromática R- 431 No. 751 escala 1:20.000 de 1957. IGAC, Proyección Gauss - Kruger, Colombia (Transversal de Mercator), con origen Bogotá, 1980. Tunja, agosto, 2012. 


\section{Condiciones naturales de la región de Colonización en 1977, Cimitarra, Santander, Colombia, escala 1:10.000}

Los datos presentados, según la aerofotografía de 1977, a escala 1:10.000, en la figura 3, en la ubicación del centro de colonización, es decir, en el área de Cimitarra, patentizan la disminución del área de bosque (de 73,89\% en 1950 a 23,12\% en 1977) y el incremento en potrero (de $18,50 \%$ en 1950 a 65,62\% en 1977), lo cual se debe a la explotación maderera generada a partir del proceso de colonización orientada en 1953, por el gobierno del general Gustavo Rojas Pinilla, potrero que fue utilizado para nuevas vocaciones económicas en agricultura y ganadería, actividades que tuvieron lugar especial en el programa de colonización dirigida. Entonces, como se pudiera observar en otras experiencias colonizadoras, la potrerización se convierte en una de las tendencias características en la posesión y uso del suelo en Colombia.

Tabla 3. Ocupación, uso del suelo según aerofotografía de 1977 a escala 1:10.000, Cimitarra - Carare, Santander, Colombia

\begin{tabular}{|l|c|c|}
\cline { 2 - 3 } \multicolumn{1}{c|}{} & Área $(\mathrm{m} 2)$ & $\%$ \\
\hline Bosque & 1516222,28 & 23,12 \\
\hline Potrero (pastizales) & 4303034,67 & 65,62 \\
\hline Recurso hídrico & 382902,49 & 5,84 \\
\hline Área Urbana & 355258,08 & 5,42 \\
\hline Total & 6557417,53 & 100 \\
\hline
\end{tabular}

Fuente: Autora, con base en la aerofotografía de 1977, a escala 1:10.000. Tunja, agosto, 2012.

Figura 3. Cobertura uso del suelo Cimitarra - Carare, Santander, Colombia, 1977

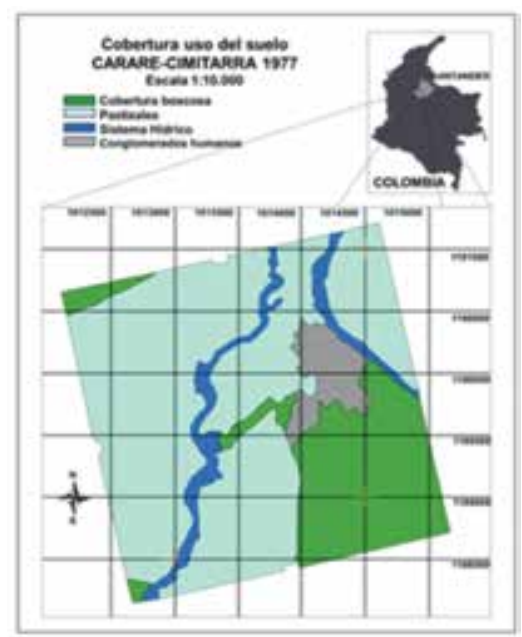

Fuente: Autora. Cobertura uso de suelo, Cimitarra - Carare, Santander, Colombia, 1977. Aerofotografía pancromática C-1757 No. 00 escala 1:10.000 de 1977. IGAC, Proyección Gauss - Kruger, Colombia (Transversal de Mercator), con origen Bogotá, 1980. Tunja, agosto, 2012. 
La colonización indujo la potrerización de áreas ricas en bosques biodiversos; originó cambios drásticos en el paisaje; dio lugar a procesos migratorios, originó cambios en el estilo de vida de los colonos ubicados en la región del Carare; generó cambios en los hábitos de consumo; incrementó la demanda de bienes y servicios; ocasionó relocalización de la población; generó ruptura de lazos de identidad y arraigo; suscitó disputas por territorios; ocasionó disputas por recursos naturales; intensificó las actividades extractivas; generó cambios en el uso del suelo; originó cambios en las relaciones sociales de producción entre otros impactos negativos. A través de la historia, los procesos de colonización han implicado la transformación y alteración del entorno rural, particularmente lo relativo al paisaje, a la diversidad de la flora y la fauna nativas, a las fuentes hídricas y a los conglomerados de poblamiento.

Este evento se ve reflejado en los cuadros siguientes, que identifican la transformación del paisaje en la región de Cimitarra, de 1950 a 1977, con una enorme pérdida de diversidad vegetal y animal del entorno, probablemente con la desaparición total o parcial de muchas especies, que posiblemente no se encontrarán en otro territorio, debido a que Colombia y gran parte de sus bosque son considerados Hotspots $o$ sitios de alta diversidad biológica y que requieren de programas especiales de conservación.

En el terreno de la biodiversidad, los programas especiales plantean una mayor explotación de los recursos forestales distintos a la madera. Estos incluyen una variada gama que va desde las frutas, nueces y especias hasta las plantas con propiedades medicinales (se estima que las plantas tropicales suministran los ingredientes activos para más o menos la mitad de las prescripciones médicas de todo el mundo), vegetales con propiedades para elaborar cosméticos y fibras para vestidos.

Actualmente en Indonesia unas 200000 personas se dedican a cosechar roten (una especie de bejuco), lo que equivale al triple de las personas empleadas para explotar madera, con ventas aproximadas a los 3 mil millones de dólares. La venta de productos forestales, diferentes a la madera, subió de un 3\% del valor total de exportaciones forestales en 1973 a un 15\% en la década del ochenta (Saavedra, 2001).

Al alterarse un ecosistema, todos sus componentes se ven afectados, sin embargo, en la región de Cimitarra, la transformación de la zona de bosque fue intensa y más acelerada mientras que, el recurso hídrico se vio más lentamente afectado, probablemente debido a que es costumbre de los procesos de colonización respetar los bosques riparios, o bosques de borde de cuerpos de aguas, para que sirvan de amortiguación de nacederos y de agua que requiere la comunidad; sin embargo, la afectación de la vegetación implica que se afecte directa o indirectamente la vegetación y fauna acompañante de las riberas de los ríos.

En la tabla 1 se evidencia que la transformación del paisaje ha sido significativa en la región de Cimitarra, Carare, debido al impacto que el hombre ejerció en los procesos de entresaca, tala selectiva, generación de matrices de pastos con fines 
de ganadería y alteración del ecosistema original para la agricultura, de tal manera que dicho impacto diezmó los bosques prístinos (bosques originales) en el área de estudio. Dichos eventos también influyeron en la modificación del clima, y los niveles de humedad relativa y ambiental se vieron afectados, dada la disminución de la capa vegetal que es impactada por la acción de las lluvias, del viento y del sol.

\section{Consolidado zona de bosque, potrero, zona hídrica y conglomerado humano región de Cimitarra - Carare, Santander, Colombia, escala 1:10.000}

Tabla 4. Ocupación-uso del suelo según aerofotografía de 1977 a escala 1:10.000, Cimitarra - Carare, Santander, Colombia

\begin{tabular}{cc}
\hline Años & Bosque (\% de área) \\
\hline 1950 & 74 \\
1957 & 32 \\
1977 & 23 \\
\hline & Fuente: Autora, 2012.
\end{tabular}

Gráfica 1. Porcentaje zona de bosque en la región de Cimitarra - Carare, Santander, Colombia

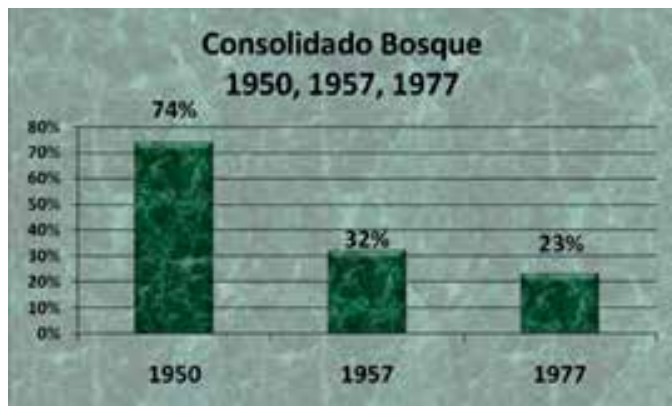

Fuente: Autora con base en la tabla 4

Tabla 5. Consolidado zona de potrero región de Cimitarra - Carare, Santander, Colombia, escala 1:10.000

\begin{tabular}{cc}
\hline Años & Potrero (\% de área) \\
\hline 1950 & 19 \\
1957 & 30 \\
1977 & 66 \\
\hline
\end{tabular}

Fuente: Autora, 2012. 
Gráfica 2. Porcentaje zona potrerizada en la región de Cimitarra-Carare, Santander, Colombia

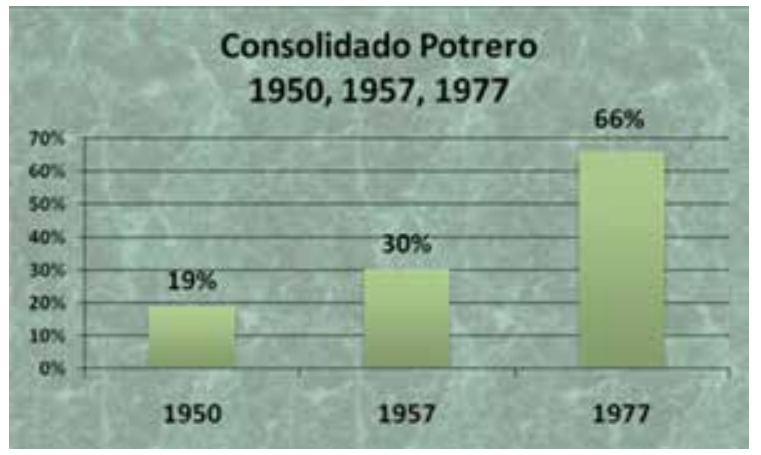

Fuente: Autora con base en el cuadro 5.

Tabla 6. Consolidado zona hídrica región del Cimitarra - Carare, Santander, Colombia, escala 1:10.000

\begin{tabular}{cc}
\hline Años & Recurso hídrico (\% de área) \\
\hline 1950 & 8 \\
1957 & 6 \\
1977 & 6 \\
\hline
\end{tabular}

Fuente: Autora, 2012.

Gráfica 3. Porcentaje recurso hídrico en la región de Cimitarra-Carare, Santander, Colombia

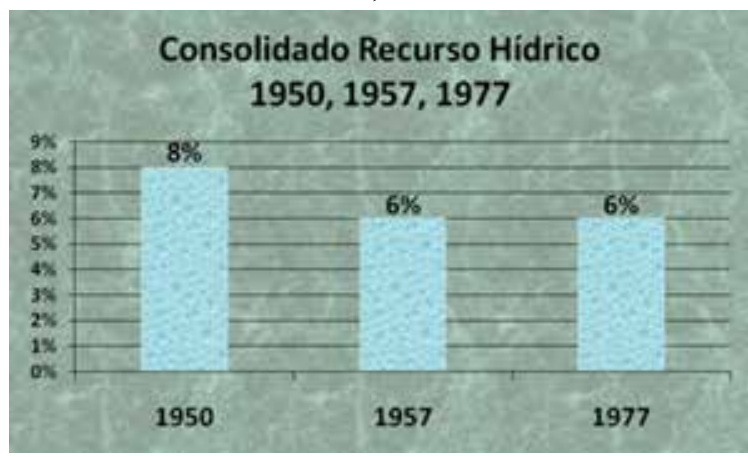

Fuente: Autora con base en la tabla 6. 
Tabla 7. Consolidado conglomerado humano Cimitarra - Carare, Santander, Colombia, escala 1:10.000

\begin{tabular}{cc}
\hline Años & Area Urbana (\%) \\
\hline 1950 & 0 \\
1957 & 1 \\
1977 & 5 \\
\hline
\end{tabular}

Fuente: Autora, 2012.

Gráfica 4. Consolidado área urbana en la región de Cimitarra-Carare, Santander, Colombia

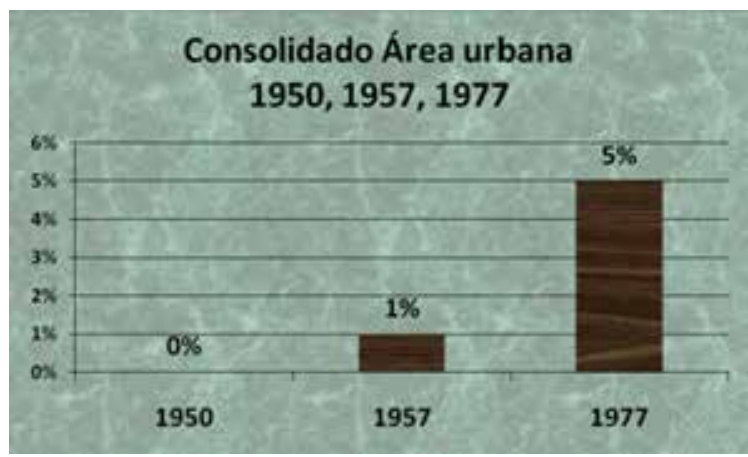

Fuente: Autora con base en la tabla 7.

Teniendo en cuenta el consolidado de la zona de potrero en el cuadro 10, se evidencia la transformación de Cimitarra - Carare, al pasar de $19 \%$ de potrero en 1950 a $30 \%$ en 1957 y $66 \%$ en 1977; debido al establecimiento de las fábricas de explotación maderera y a la construcción de obras de infraestructura ejecutadas por la institucionalidad que hizo presencia en la región a partir del programa de colonización orientado por el Gobierno nacional en el periodo 1953-1957.

\section{Conclusiones}

La política de colonización agenciada por el general Gustavo Rojas Pinilla en (1953-1957) forjó transformaciones en el paisaje, amplió la frontera agraria interior y generó una intensa movilidad poblacional hacia las zonas de colonización como la región del Carare.

El establecimiento de la empresa colonizadora provocó diversas formas de poblamiento sobre la región, el reclutamiento de trabajadores para la empresa colonizadora encargados de la labor de desmonte maderero; producto de este proceso 
se genera un intenso deterioro del bosque y la emergencia de la potrerización de amplios espacios en la región.

La desaparición de numerosos riachuelos y pequeños afluentes que enriquecían las principales vertientes hídricas y ciénagas de la región del Carare y, por consiguiente, el área hídrica disminuyó notablemente.

El desarrollo de esta investigación presenta a la comunidad académica y científica un modelo novedoso de medición de impacto ambiental de los procesos de colonización de frontera interior. A partir de la caracterización de mapas y aerofotografías del periodo de estudio y de su contexto multitemporal, se evidencia la profunda y acelerada transformación del paisaje en la región.

Se ubicaron y seleccionaron en el IGAC, las cartas correspondientes a los años 1941, 1952 y 1972, las cuales se utilizaron como referente para situar la zona del Carare, teniendo en cuenta las coordenadas institucionales del IGAC, y se produjeron tres nuevos mapas, fruto de esta investigación, de la región priorizada por el general Gustavo Rojas Pinilla, para establecer el programa de colonización del periodo 1953-1957. Los nuevos mapas evidenciaron cambios de paisaje en cuanto a bosques, recurso hídrico, carreteables, caminos construidos y en vía de construcción, límites geográficos y sitios de poblamiento en la región, estos cambios producto del programa de colonización se observaron en la construcción del nuevo mapa de 1972.

Se logró llevar a escala 1:10.000 las tres aerofotografías seleccionadas, con el propósito de superponerlas y compararlas sobre una misma zona; para ello se seleccionó el sitio de ubicación de la población de Cimitarra, sede del programa de Colonización del Carare, se midieron las áreas boscosa, de potrero, hídrica y urbana en las tres épocas, y se determinó con alto grado de exactitud el profundo impacto ambiental negativo que generó el establecimiento de colonos.

Se puede concluir que la empresa colonizadora creada para la región del Carare, por el gobierno del general Rojas Pinilla, produjo la eliminación o el desplazamiento, hacia otras zonas de la geografía nacional de especies nativas de flora y fauna; pero también, propició una nueva colonización de especies animales y vegetales que llegaron junto con la colonización humana a la región del Carare, muchas de ellas en condiciones migratorias, y otras traídas por el hombre, como especies domésticas o en proceso de domesticación, lo cual debe ser objeto de futuras investigaciones ambientales. Esta situación implicó el cambio de la dinámica de los ecosistemas; es el caso de modelos agroforestales o silvopastoriles implementados por los colonos para surtir sus necesidades alimentarias, medicinales y de especies ornamentales, entre otras.

Esta clase de expansión agrícola afectó notablemente el medioambiente, lo cual se evidenció en el tamaño de los bosques que se limpiaron para convertirlos en potreros; se dio además, un acelerado proceso de valorización del suelo, lo cual generó otras relaciones de producción típicamente capitalistas, tales como: concentración de la propiedad y trabajo asalariado. 
Se da respuesta a la pregunta formulada en el proyecto de investigación: ¿Contribuyó el programa de colonización agraria del periodo de gobierno 1953-1957 a ampliar la frontera agrícola del Carare, Santander en Colombia, de manera diferente a otras formas contemporáneas de colonización agraria? A pesar del gran impacto ambiental negativo, la frontera agraria se amplió con fines económicos y sociales en aras de superar el conflicto político y social característico de la época en Colombia.

El modelo novedoso de medición de impacto ambiental a partir de un programa colonizador, que se presenta en este artículo, se puede utilizar en Colombia y en el mundo, con el fin de medir impactos ambientales, que pretenden justificarse en términos económicos y sociales, dados sus objetivos de vincular a la economía nacional e internacional, potenciales productivos que apoyarían el crecimiento y desarrollo de otras regiones o países, como es el caso de la Orinoquía colombiana.

\section{Referencias}

Cortez, C. (1991). Geografía histórica. México: UAM, Instituto Mora. Consejo Nacional de Universidades, Oficina de Planificación del Sector Universitario.

Cunill, P. (2009). Historia de la geografía de Venezuela: siglos XV-XX (vol. 2).

DANE et al. (1952). Archivo cartográfico estadística nacional. Bogotá.

García, B. (1998). En busca de la geografía histórica. Relaciones, estudios de historia y sociedad, historia y geografía, 75(XIX), 25-58.

García, O. M. (2012). Colonización del Carare Santander Colombia 1953- 1957 [Tesis doctoral]. Universidad Pedagógica y Tecnológica de Colombia, Tunja.

IGAC. (1941). Carta geográfica del departamento de Santander. Bogotá: Instituto Geográfico Agustín Codazzi: Oficina de Longitudes.

IGAC. (1950). Aerofotografía pancromática C-584 No. 172 escala 1:30.000. Bogotá: Instituto Geográfico Agustín Codazzi.

IGAC. (1957). Aerofotografía pancromática R-431 No. 751 escala 1:20.000. Bogotá: Instituto Geográfico Agustín Codazzi.

IGAC. (1977). Aerofotografía pancromática C-1757 No. 00 escala 1:20.000. Bogotá: Instituto Geográfico Agustín Codazzi.

IGAC. (1989). Atlas de Colombia. Bogotá: Instituto Geográfico Agustín Codazzi. Ministerio de Hacienda y Crédito Público e IGAC. (1972). Carta preliminar. Bogotá.

Molano, J. (2004). Villa de Leyva: Ensayo de interacción social de una catástrofe ecológica. Biblioteca Virtual del Banco de la República. 
Palacios, M. (2011). ¿De quién es la tierra? Bogotá: Fondo de Cultura Económica Ltda., Universidad de los Andes.

Presidencia de la República. (1953). Decreto 0870 del 26 de marzo. Bogotá: Diario Oficial.

Prince, H. (1985). La geografía histórica en 1980.

Saavedra, L. E. (2001). Impacto de los megaproyectos de infraestructura vial sobre la biodiversidad. Quinto Taller: Comercio y Valoración de la Biodiversidad e Impacto de Megaproyectos de Infraestructura. Bogotá: Banco Interamericano de Desarrollo, Secretaría General de la CAN, Ministerio de Ambiente de Colombia, Consorcio GTZ/FUNDECO/Instituto de Ecología.

Sauer, C. O. (1925). The Morphology of Landscape. California: Berkeley.

\section{Infografía}

www.banrepcultural.org/blaavirtual/geografia/vleiva/p249-254.htm 